\title{
REDUNDANCY RELATIONS FOR FAULT DIAGNOSIS IN NONLINEAR UNCERTAIN SYSTEMS
}

\author{
ALEXEY SHUMSKY
}

Institute for Marine Technology Problems

Far Eastern Branch of the Russian Academy of Sciences

Sukhanova 5a, Vladivostok, 690950, Russia

e-mail: shumsky@mail.primorye.ru

\begin{abstract}
The problem of fault detection and isolation in nonlinear uncertain systems is studied within the scope of the analytical redundancy concept. The problem solution involves checking the redundancy relations existing among measured system inputs and outputs. A novel method is proposed for constructing redundancy relations based on system models described by differential equations whose right-hand sides are polynomials. The method involves a nonlinear transformation of the initial system model into a strict feedback form. Algebraic and geometric tools are used for this transformation. The features of the method are made particular for uncertain systems with a linear structure.
\end{abstract}

Keywords: fault detection and isolation, nonlinear systems, redundancy relations, full decoupling, algebraic approach, geometric approach.

\section{Introduction}

An increasing demand for reliability, fault tolerance and safety in critical purpose systems stimulates the use of fault detection and isolation (FDI) methods. Numerous methods for on-line FDI have been proposed within the framework of analytical redundancy (Chow and Willsky, 1984). According to this concept, FDI is based on checking relations that exist among system inputs and outputs measured over a finite time window. The FDI process includes residual generation as a result of the mismatch between the system behaviour and its reference model behaviour, followed by decision making through evaluation of the residual. Open-loop and closed-loop techniques have been elaborated for solving the residual generation problem including redundancy or parity relations (Chow and Willsky, 1984; Comtet-Varga et al., 1999; Gertler and Kunwer, 1993; Medvedev, 1994; Mironovskii, 1980; Lou et al., 1986; Shumsky, 2002) as well as diagnostic and adaptive observers (De Persis and Isidori, 2001; Ding and Frank, 1993; Patton and Kangethe, 1989; Seliger and Frank, 1991).

This paper concentrates on the problem of constructing redundancy relations. Its solution usually deals with the task of eliminating unknown system states. First solutions of the elimination task with application to construc- ting redundancy relations were proposed for linear systems by Mironovskii (1980) and then by Chow and Willsky (1984). In (Comtet-Varga et al., 1999), the problem of constructing nonlinear redundancy relations was considered for continuous-time polynomial systems. In the framework of the above paper, special tools were considered for solving the state elimination task involving elimination theory (Diop, 1991), Groebner bases (Cox et al., 1992) and characteristics sets (Ritt, 1950). The advantage of the proposed solution is the possibility to use various software packages (e.g., the Groebner package in the computer algebra system Maple) to support the calculations. The drawback of the proposed solution is time differentiation of the measured system inputs and outputs that fails if measurement noise is present. Another method was proposed by Medvedev (1994). In contrast to the above work, this method uses integrating the measured system inputs and outputs, which results in smoothing the noise effect. But the last method is applicable only to linear systems.

As soon as the modelling uncertainty and faults both act upon the residual, the robustness problem arises. The essence of robust FDI is to make the residual insensitive to uncertainty and, simultaneously, sensitive to faults. There exist three main approaches to solve the robustness problem. The first of them is based on the adaptation princi- 
ple. Its use assumes that the uncertainties represented as unknown constant (or slow varying) coefficients of the reference model are estimated for tuning the reference model (Ding and Frank, 1993; Isermann, 1993; Shumsky 2002). A realization of this approach may cause difficulties if the number of the unknown coefficients subjected to estimation is considerable.

The second approach is closely related to the full decoupling problem whose solutions were considered in several works (De Persis and Isidori, 2001; Frank, 1990; Massoumnia, 1986; Massoumnia et al., 1989; Patton and Kangethe, 1989; Seliger and Frank, 1991; Shumsky, 1991; Shumsky and Zhirabok, 2006). Note that all these solutions involve no demands on the time behaviour of unknown coefficients but are characterized by the existence conditions that impose strong limitations on the acceptable number of the above coefficients.

The third approach deals with the optimization principle. Various criteria were proposed in the framework of the multi-objective optimization problem aimed at achieving a compromise between the low sensitivity of the residual to uncertainty and its high sensitivity to the faults (Chow and Willsky, 1984; De Persis and Isidori, 2002; Gertler and Kunwer, 1993; Lou et al., 1986). By increasing the acceptable number of unknown coefficients, this approach rejects the idea to make the residual insensitive to uncertainty.

An interesting solution of the robust residual generation problem was proposed by Pekpe et. al. (2004) for sensor FDI in stable systems with a linear structure. A distinguishing feature of this solution is that it does not use the explicit model: all the matrices of the linear model are assumed to be unknown. Thus, it allows us to overcome the limitations of the conventional full decoupling based methods. An advantage of the last solution is that it gives rise to a new view on the robustness problem.

In this paper, a novel method is proposed for constructing the redundancy relations for robust FDI in nonlinear uncertain systems described by differential equations whose right-hand sides are polynomials. In order to concentrate on the main result, the problem statement is deliberately simplified. Firstly, only the noise free case is considered. Of course, it does not completely correspond to a real situation but it will allow us to exclude the repetition of known results dealing with optimization techniques, filtering, statistical data handling and threshold based decision logic which are not immediately related to the method. Note that the solution proposed below assumes integrating the measured system inputs and outputs, which results in smoothing the noise effect. Secondly, as soon as the hypothesis on the absence of noise is made, the decision making procedure will consist in the comparison of the residual vector components with zero.

The method proposed in the paper involves a nonlinear transformation of the initial system model into a strict feedback form and, then, its conversion to an input-output description. Following (Pekpe et al. 2004), the redundancy relations are immediately found from the last description. In order to isolate the faults, a full decoupling based method (Shumsky and Zhirabok, 2006) is used to obtain structural residuals. The result of the last paper can be also applied to fault detectability and isolability analysis.

Special attention in the paper is paid to nonlinear transformation of the system model into a strict feedback form because such a transformation forms a basis for the proposed method. The existence conditions for transforming and designing the algorithm were formulated by Shumsky (1992) in the framework of the algebraic approach (the algebra of functions). In the present paper, taking into account the links between the algebra of functions and the differential geometric approach indicated by Shumsky and Zhirabok (2006), these conditions and the algorithm are reformulated in geometric terms. The aim is to obtain a simplified computer aided procedure for a transformation.

To make a comparison between new and known (Pekpe et al., 2004) results, the proposed method is substantiated for systems with a linear structure. Also, to demonstrate the application of the proposed method to nonlinear systems, a nonlinear simulation example is given.

The paper is organized as follows: In Section 2, the FDI method is described. Section 3 is devoted to the problem of the nonlinear model transformation. The linear system case is considered in Section 4. The nonlinear example is given in Section 5. Section 6 concludes the paper.

\section{Description of the FDI Method}

Let the system under diagnosis be specified by differential equations of the form

$$
\begin{aligned}
& x(t)=f(x(t))+g(x(t)) u(t)+d(x(t)) w(t), \\
& y(t)=h(x(t)),
\end{aligned}
$$

where $x(t) \in X \subseteq \mathbb{R}^{n}$ is the state vector, $u(t) \in U \subseteq \mathbb{R}^{p}$ is the input vector, $y(t) \in Y \subseteq \mathbb{R}^{l}$ is the output vector, $w(t) \in \mathbb{R}^{q}$ is the vector given for fault representation, $f, h$ and $g, d$ are nonlinear vector and matrix functions, respectively. It is assumed that all the components of the functions $f, h, g$ and $d$ have a polynomial form, i.e.,

$$
\begin{gathered}
P_{\vartheta}(x)=\sum_{i} \vartheta_{i} x_{1}^{\sigma_{1, i}} x_{2}^{\sigma_{2, i}} \ldots x_{n}^{\sigma_{n, i}}, \\
\sigma_{j, i}, \quad 1 \leq j \leq n
\end{gathered}
$$

with constant coefficients $\vartheta_{i} \in \mathbb{R}$. It is also assumed that some or all coefficients are unknown. Note that (3) is used below as the general form for the polynomial representation. 
Let $w(t)=0$ hold for a healthy system. In fault conditions we have $w(t) \neq 0$ and $w_{j}(t) \neq 0$ corresponds to the $j$-th fault. Under this assumption, as soon as no assumption is made about fault dynamics, $w_{j}(t)$ is considered an arbitrary function of time. Notice that given a representation of the faults corresponds not only to actuator or plant faults, but also to sensor faults, considered as pseudoactuator faults (Massoumnia et al., 1989; Park et al., 1994).

For the system (1), (2) consider a way for constructing redundancy relations. Let $w(t)=0$ and introduce the coordinate transformation

$$
\begin{aligned}
x_{*}^{(i)}(t) & =\alpha^{(i)}(x(t)), \quad 1 \leq i \leq k, \\
y_{*} & =\phi(y(t)),
\end{aligned}
$$

such that in the transformed coordinates the system (1), (2) takes the strict feedback form

$$
\begin{aligned}
x_{*}^{(1)}(t)= & f_{*}^{(1)}(y(t))+g_{*}^{(1)}(y(t)) u(t), \\
x_{*}^{(i)}(t)= & f_{*}^{(i)}\left(x_{*}^{(1)}(t), \ldots, x_{*}^{(i-1)}(t), y(t)\right) \\
& +g_{*}^{(i)}\left(x_{*}^{(1)}(t), \ldots, x_{*}^{(i-1)}(t), y(t)\right) u(t), \\
y_{*}(t)= & h_{*}\left(x_{*}^{(1)}(t), \ldots, x_{*}^{(k)}(t)\right) .
\end{aligned}
$$

The structural interpretation of the strict feedback form is given in Fig. 1, where $S^{(i)}$ is the feedback-less dynamic subsystem specified by an appropriate equation from (6) and index $k$ corresponds to the number of subsystems included into this form.

The components of $\alpha^{(i)}$ and $\phi$ are assumed to be polynomials of the forms $P_{\vartheta}(x)$ and $P_{\vartheta}(y)$, while the components of $f_{*}^{(i)}, g_{*}^{(i)}$ and $h_{*}$ take the polynomial forms $P_{\vartheta}\left(\chi^{(i-1)}\right)$ and $P_{\vartheta}\left(\chi^{(k)}\right)$, respectively, where $\chi^{(i-1)}=$ $\left(x_{*}^{(1)^{T}}, \ldots, x_{*}^{(i-1)^{T}}, y^{T}\right)^{T}, \chi^{(k)}=\left(x_{*}^{(1)^{T}}, \ldots, x_{*}^{(k)^{T}}\right)^{T}$. It is a principle in further deliberations if, in contrast to the functions $\alpha_{(i)}, f_{*}^{(i)}, g_{*}^{(i)}$ and $h_{*}$, the function $\phi$ does not contain unknown coefficients.

For the system (6) and all $i, 1 \leq i \leq k$, the corresponding iterated integral is a functional of system inputs and outputs defined on the time interval $\left[t, t_{*}\right]$ by the recurrence

$$
\begin{aligned}
& I_{1}\left(t, t_{*}\right) \\
& =\int_{t}^{t_{*}}\left(f_{*}^{(1)}\left(y\left(\tau_{1}\right)\right)+g_{*}^{(1)}\left(y\left(\tau_{1}\right)\right) u\left(\tau_{1}\right)\right) \mathrm{d} \tau_{1}+x_{*}^{(1)}(t), \\
& I_{i}\left(t, t_{*}\right) \\
& =\int_{t}^{t_{*}}\left(f_{*}^{(1)}\left(I_{1}\left(t, \tau_{i}\right), \ldots, I_{(i-1)}\left(t, \tau_{i}\right), y\left(\tau_{i}\right)\right)\right. \\
& \left.\quad+g_{*}^{(1)}\left(I_{1}\left(t, \tau_{i}\right), \ldots, I_{(i-1)}\left(t, \tau_{i}\right), y\left(\tau_{i}\right)\left(\tau_{i}\right)\right)\right) \mathrm{d} \tau_{i} \\
& \quad+x_{*}^{(i)}(t) .
\end{aligned}
$$

From (6) and (8) it is immediately seen that

$$
x_{*}^{(i)}\left(t_{*}\right)=I_{i}\left(t, t_{*}\right) .
$$

Taking (7) and (9) into account, we obtain

$$
y_{*}\left(t_{*}\right)=h_{*}\left(I_{1}\left(t, t_{*}\right), \ldots, I_{k}\left(t, t_{*}\right)\right) .
$$

Because of the polynomial form for all functions from (6) and (7), Eqn. (10) can be rewritten as follows:

$$
y_{*}\left(t_{*}\right)=C(t) W\left(t, t_{*}\right),
$$

where the matrix $C(t)$ dependent on unknown coefficients and unknown vectors $x_{*}^{(1)}(t), \ldots, x_{*}^{(k)}(t)$ is time invariant on the time interval $\left[t, t_{*}\right]$, while $W\left(t, t_{*}\right)$ is the column vector of functionals dependent only on the system inputs and outputs measured at this time interval. Let $t_{i}=t+$ $i_{\Delta} t$, where $\Delta t$ is some sampling period, and introduce the matrix $V_{i}\left(t_{i}\right)=\left(W(t, t) W\left(t, t_{1}\right) \ldots W\left(t, t_{i}\right)\right)$. Denote by $s$ the minimal integer satisfying the condition

$$
\operatorname{rank} V_{s}\left(t_{s}\right)=\operatorname{rank} V_{s-1}\left(t_{s-1}\right) .
$$

Note that $s-1$ does not exceed the number of linear independent components of $W\left(t, t_{*}\right)$. Write $Y\left(t_{s}\right)=$ $\left(y_{*}(t) y_{*}\left(t_{1}\right) \ldots y_{*}\left(t_{s}\right)\right)$. According to (11), the equality $Y\left(t_{s}\right)=C(t) V_{s}\left(t_{s}\right)$ holds. According to (12), the matrix $V_{s}\left(t_{s}\right)$ is singular. Using a nonzero vector

$$
v\left(t_{s}\right) \in \operatorname{ker} V_{s}\left(t_{s}\right),
$$

from the above one obtains $Y\left(t_{s}\right) v\left(t_{s}\right)=0$ independently of the value of the unknown matrix $\mathrm{C}(\mathrm{t})$. Finally, the computational form for robust redundancy relations is given by

$$
r\left(t_{s}\right)=\Phi_{s}\left(t_{s}\right) v\left(t_{s}\right)
$$

with the matrix

$$
\Phi_{s}\left(t_{s}\right)=\left(\phi(y(t)) \phi\left(y\left(t_{1}\right)\right) \ldots \phi\left(y\left(t_{s}\right)\right)\right)
$$

involved instead of the matrix $Y\left(t_{s}\right)$. As soon as the matrix $V_{s}\left(t_{s}\right)$ and the vector $v\left(t_{s}\right)$ can be calculated online by handling system inputs and outputs measured on the time interval $\left[t, t_{s}\right]$, the knowledge of system inputs and outputs over this time interval is all one needs to calculate the residual $r\left(t_{s}\right)$ at instant $t_{s}$. To generate the residual according to (13), we need expressions for the function $\phi$ and the vector $W\left(t, t_{s}\right)$ which should be obtained off-line.

Let us make clear the conditions under which the redundancy relations of the form (13) can be applied to fault detection and isolation. Consider the fault detection problem first. Assume that a fault in the system, appearing within the time interval $\left[t, t_{s}\right]$, results in a distortion of the 


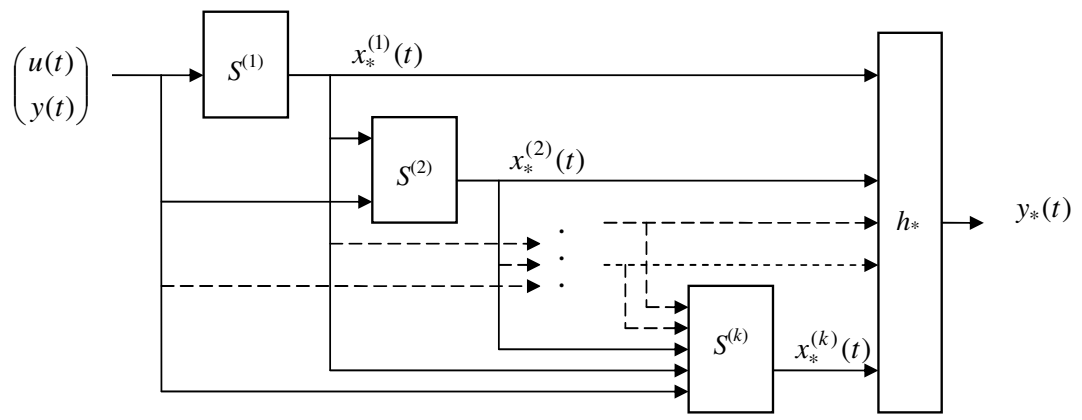

Fig. 1. Structure interpretation of the strict feedback form.

system output. Due to the polynomial forms for all functions from (1), (2), (4)-(7), we may write

$$
y_{*}\left(t_{*}\right)=(C(t) E(t))\left(\begin{array}{c}
W\left(t, t_{*}\right) \\
\Psi\left(t, t_{*}\right)
\end{array}\right),
$$

where the matrix $E(t)$ is time invariant on the time interval $\left[t, t_{*}\right]$ and $\Psi\left(t, t_{*}\right)$ is the column vector of functionals dependent on the system inputs and outputs as well as on unknown vectors of the state and faults. Therefore, in the faulty case we have

$$
r\left(t_{s}\right)=E(t)\left(\Psi(t, t) \Psi\left(t, t_{1}\right) \ldots \Psi\left(t, t_{s}\right)\right) v(t-s) .
$$

Under the assumption

$$
\left(\Psi(t, t) \Psi\left(t, t_{1}\right) \ldots \Psi\left(t, t_{s}\right)\right) v(t-s) \neq 01,
$$

the necessary condition for fault detection follows immediately from (15):

$$
E(t) \neq 0 .
$$

The condition (16) fails if

$$
\frac{\partial \alpha^{(i)}(x)}{\partial x} d_{j}(x)=0, \quad 1 \leq i \leq k
$$

for each column $d_{j}(x), 1 \leq j \leq q$ of the matrix $d(x)$, because in this case the functions $\alpha^{(i)}, 1 \leq i \leq k$ define a transformation of (1) and (2) to (6) and (7) and, therefore, the representation (11) holds even if $w(t) \neq 0$ (see Remark 1 in the next section for details).

To isolate faults, a bank of redundancy relations is involved. Every redundancy relation from this bank is used to generate the appropriate subvector of the residuals $r^{(m)}, m=1,2, \ldots, N$, and the residual vector $r$ is composed of these subvectors. The structural properties of the residual vector are characterized by the binary matrix of fault syndromes (Table 1) with the elements $s_{m j}=0$ if the subvector $r^{(m)}$ is insensitive to the fault caused by $w_{j}(t) \neq 0$, otherwise $s_{m j}=1$.

\footnotetext{
${ }^{1}$ This assumption looks reasonable if we take into account that the components of $\Psi\left(t, t_{*}\right)$ are linear functionals independent of the functionals from $W\left(t, t_{*}\right)$.
}

To isolate a fault, it is necessary (and sufficient) that every two columns of this matrix do not coincide. Note that (17) corresponds exactly to the case when the residual is insensitive to the fault caused by $w_{j}(t) \neq 0$. Consequently, if (17) holds for some $m$ and $j$, then $s_{m j}=0$, otherwise one has $s_{m j}=1$. Taking this into account, one can obtain different alternatives for choosing the matrix of fault syndromes by an appropriate choice of the functions $\alpha^{(i)}, 1 \leq i \leq k$, according to (17).

\section{Model Transformation}

In this section, the algebra of functions is used to formulate existence conditions for a transformation into a strict feedback form. Then differential geometric tools are involved to obtain a procedure for this transformation.

3.1. Existence Conditions for the Transformation. Let $w(t)=0$ in (1) and consider the functions $\alpha^{(i)}, 1 \leq$ $i \leq k$, and $\phi$ from (4) and (5), respectively.

Lemma 1. The system (1), (2) admits a transformation into a strict feedback form (6), (7) if and only if the functions $\alpha^{(i)}, 1 \leq i \leq k$ and $\phi$ satisfy the conditions

$$
\begin{aligned}
& f^{(1)}(h(x))=\frac{\partial \alpha^{(1)}(x)}{\partial x} f(x), \\
& f^{(i)}\left(\alpha^{(1)}(x), \ldots, \alpha^{(i-1)}(x), h(x)\right) \\
&=\frac{\partial \alpha^{(1)}(x)}{\partial x} f(x), \quad 2 \leq i \leq k,
\end{aligned}
$$

Table 1. Matrix of fault syndromes.

\begin{tabular}{|c||c|c|c|c|}
\hline \multirow{2}{*}{ Residual subvector } & \multicolumn{4}{c|}{ Faults } \\
\cline { 2 - 5 } & $w_{1}(t) \neq 0$ & $w_{2}(t) \neq 0$ & $\ldots$ & $w_{q}(t) \neq 0$ \\
\hline \hline$r^{(1)}$ & $s_{11}$ & $s_{12}$ & $\ldots$ & $s_{1 q}$ \\
\hline$r^{(N)}$ & $s_{21}$ & $s_{22}$ & $\ldots$ & $s_{2 q}$ \\
\hline$\vdots$ & $\vdots$ & $\vdots$ & $\vdots$ & $\vdots$ \\
\hline$r^{(N)}$ & $s_{N 1}$ & $s_{N 2}$ & $\ldots$ & $s_{N q}$ \\
\hline
\end{tabular}




$$
\begin{aligned}
g^{(1)}(h(x)) & =\frac{\partial \alpha^{(1)}(x)}{\partial x} g(x), \\
g^{(i)}\left(\alpha^{(1)}(x), \ldots, \alpha^{(i-1)}(x), h(x)\right) & \\
& =\frac{\partial \alpha^{(1)}(x)}{\partial x} g(x), \quad 2 \leq i \leq k,
\end{aligned}
$$

and

$$
h_{*}\left(\alpha^{(1)}(x), \ldots, \alpha^{(i-1)}(x)\right)=\phi(h(x)) .
$$

The proofs of Lemma 1 and Theorem 1 are given in Appendix.

Remark 1. Let the functions $\alpha^{(i)}, 1 \leq i \leq k$ satisfy the conditions (17)-(19). In this case the system (1), (2) admits a transformation into the strict feedback form (6), (7) even if $w_{j}(t) \neq 0$. Indeed,

$$
\begin{aligned}
x_{*}^{(i)} & =\frac{\partial \alpha^{(i)}}{\partial x}\left(f(x)+g(x) u+d_{j}(x) w_{j}\right) \\
& =\frac{\partial \alpha^{(i)}}{\partial x}(f(x)+g(x) u)
\end{aligned}
$$

and the links in the proof of sufficiency hold (see Appendix).

Thus the functions $f_{*}^{(i)}, g_{*}^{(i)}, 1 \leq i \leq k$ and $h_{*}$ are found from (18) and (19) given functions $\alpha^{(i)}, 1 \leq i \leq k$ and $\phi$. To make a step to find the functions $\alpha^{(i)}, 1 \leq i \leq k$ and $\phi$, the solvability condition for Eqns. (18) and (19) is formulated in algebraic terms. In order to overcome possible obstacles, the definitions of algebraic tools in use precede this formulation. If necessary, more detailed information about these tools can be found in (Shumsky and Zhirabok, 2006).

Denote by $\Im_{X}$ the set of vector functions with a domain $X$. For vector functions from $\Im_{X}$ define a partial preordering relation as a binary operation, as well as redefine a special binary relation for a class of linear-in-control dynamic systems of the form (1).

Definition 1. (Partial preordering relation) For $\alpha, \beta \in \Im_{X}$ the relation $\alpha \leq \beta$ holds if and only if there exists a function determined on the set of values of $\alpha$ such that $\beta=\gamma \circ \alpha$, where 'o' signifies the composition.

Definition 2. (Binary operation) For $\alpha, \beta \in \Im_{X}$ the vector function $\alpha \times \beta \in \Im_{X}$ is such that $\alpha \times \beta \leq \alpha, \alpha \times \beta \leq \beta$, and for every function $\delta \in \Im_{X}$ satisfying $\delta \leq \alpha$ and $\delta \leq \beta$ the functional inequality $\delta \leq \alpha \times \beta$ holds.

Definition 3. (Binary relation $\Delta \subset \Im_{X} \times \Im_{X}$ ) For $\alpha, \beta \in$ $\Im_{X}$ and the functions $f$ and $g$ of the system (1) $(\alpha, \beta) \in$ $\Delta$ if and only if $\alpha \leq(\partial \beta / \partial x) f, \alpha \leq(\partial \beta / \partial x) g_{j}, 1 \leq$ $j \leq p$ where $g_{j}$ constitutes the appropriate column of the matrix function $g$.
Theorem 1. Equations (18) and (19) are solvable if and only if

$$
\begin{aligned}
& \left(h, \alpha^{(1)}\right) \in \Delta, \\
& \left(h \times \alpha^{(1)} \times \ldots \times \alpha^{(i-1)}, \alpha^{(i)}\right) \in \Delta, \quad 2 \leq i \leq k, \\
& \alpha^{(1)} \times \ldots \times \alpha^{(k)} \leq \phi \circ h .
\end{aligned}
$$

A general way to find the functions $\alpha^{(i)}, 1 \leq i \leq k$ follows immediately from the relations (20) and involves a step-by-step determination of these functions. In the first step one determines the function $\alpha^{(1)}$ given a function $h$ according to the inclusion $\left(h, \alpha^{(1)}\right) \in \Delta$. In each next step $i=2,3, \ldots$, one determines the function $\alpha^{(i)}$ according to the inclusion $\left(h \times \alpha^{(1)} \times \alpha^{(i-1)}, \alpha^{(i)}\right) \in \Delta$ given a function $h$ and the functions $\alpha^{(j)}, 1 \leq j \leq i-1$ determined in the previous steps. The procedure is accomplished if for some $k$ one has

$$
\alpha^{(1)} \times \ldots \alpha^{(k)} \leq \alpha^{(k+1)},
$$

i.e., all the components of $\alpha^{(k+1)}$ are functionally dependent on the components of the function $\alpha^{(1)} \times \ldots \times \alpha^{(k)}$. To find the functions $\alpha^{(i)}, 1 \leq i \leq k$ from the appropriate inclusions and, then, the function $\phi$ from (21), differential geometric tools (Isidori, 1989) are involved.

3.2. Procedure for the Transformation. Let $\Lambda_{\alpha}$ denote the distribution introduced for a vector function $\alpha$ :

$$
\Lambda_{\alpha}=\operatorname{span}\left\{\lambda_{\alpha} \mid(\partial \alpha / \partial x) \lambda_{\alpha}=0\right\}
$$

and let the appropriate codistribution be $\Omega_{\alpha}=\Lambda_{\alpha}^{\perp}$, where the symbol ' $\perp$ ' is used for the annihilator. Recall that one may find the vector function by integrating some distribution iff this distribution is involutive with respect to the Lie brackets. For vector functions $\alpha$ and $\beta$ the connection between algebraic and differential geometric tools is given as follows: $\alpha \leq \beta$ iff $\Omega_{\alpha} \supseteq \Omega_{\beta}$ and $\Omega_{\alpha \times \beta}=\Omega_{\alpha}+\Omega_{\beta}$ (Shumsky and Zhirabok, 2006). From the above it can be easily seen that $\alpha \leq \beta$ iff $\Lambda_{\alpha} \subseteq \Lambda_{\beta}$ and the inclusion $\Lambda_{\alpha \times \beta} \subseteq \Lambda_{\alpha} \cap \bar{\Lambda}_{\beta}$ holds. Then, the expressions $h \times \alpha^{(1)} \times \ldots \times \alpha^{(i-1)}$ and (22) correspond to

$$
\operatorname{ker} \frac{\partial h}{\partial x} \cap \bigcap_{j=1}^{i-1} \Lambda_{\alpha^{(j)}}
$$

and

$$
\bigcap_{i=1}^{k} \Lambda_{\alpha^{(i)}} \subseteq \Lambda_{\alpha^{(k+1)}},
$$

respectively.

Consider the function $\delta=\phi \circ h$. It is clear that $h \leq \delta$. From the above and (21), the inclusions

$$
\operatorname{ker} \frac{\partial h}{\partial x} \subseteq \Lambda_{\delta}, \quad \bigcap_{i=1}^{k} \Lambda_{\alpha^{(i)}} \subseteq \Lambda_{\delta}
$$


follow and, as a result,

$$
\operatorname{ker} \frac{\partial h}{\partial x}+\bigcap_{I=1}^{k} \Lambda_{\alpha^{(j)}} \subseteq \Lambda_{\delta} .
$$

Consider a geometric interpretation of the relation $(\alpha, \beta) \in \Delta$. Let $\Lambda_{\beta}$ be an involutive distribution such that

$$
\Lambda_{\beta} \supseteq \operatorname{span}\left\{\left[\varphi, \lambda_{\alpha}\right], \varphi \in f, g_{1}, \ldots, g_{p}, \lambda_{\alpha} \in \Lambda_{\alpha}\right\},
$$

and

$$
L_{\varphi}\left\langle\omega_{\beta}, \lambda_{\alpha}\right\rangle=0, \quad \omega_{\beta} \in \Lambda_{\beta}^{\perp}, \quad \lambda_{\alpha} \in \Lambda_{\alpha},
$$

where the symbols $[\cdot, \cdot],\langle\cdot, \cdot\rangle$ and $L_{\varphi}\left\langle\omega_{\beta}, \lambda_{\alpha}\right\rangle$ denote the Lie brackets, the inner product and the Lie derivative of the function $\left\langle\omega_{\beta}, \lambda_{\alpha}\right\rangle$ along the vector field $\varphi$, respectively. Note that to satisfy both the relations (23) and (24), it is sufficient to take a minimal involutive distribution

$\Lambda_{\beta} \supseteq \Lambda_{\alpha}+\operatorname{span}\left\{\left[\varphi, \lambda_{\alpha}\right], \varphi \in f, g_{1}, \ldots, g_{p}, \lambda_{\alpha} \in \Lambda_{\alpha}\right\}$.

But then there is no guarantee that the obtained distribution is a minimal one satisfying (23) and (24). From the above and the well known identity (Isidori, 1989, p. 10)

$$
L_{\varphi}\left\langle\omega_{\beta}, \lambda_{\alpha}\right\rangle=\left\langle L_{\varphi} \omega_{\beta}, \lambda_{\alpha}\right\rangle+\left\langle\omega_{\beta},\left[\varphi, \lambda_{\alpha}\right]\right\rangle
$$

it follows that

$$
\left\langle L_{\varphi} \omega_{\beta}, \lambda_{\alpha}\right\rangle=0,
$$

where $L_{\varphi} \omega_{\beta}$ denotes the Lie derivative of the covector field $\omega_{\beta}$ along the vector field $\varphi$. The last equality means that $\Omega_{\alpha} \supseteq L_{\varphi} \omega_{\beta}, \varphi \in\left\{f, g_{1}, \ldots, g_{p}\right\}$. It implies $\alpha \leq(\partial \beta / \partial x) f, \alpha \leq(\partial \beta / \partial x) g_{j}, 1 \leq j \leq p$, and then $(\alpha, \beta) \in \Delta$.

Using the geometric interpretation of the algebraic relations and the operation given above, the algorithm for the determination of the functions $\alpha^{(i)}, 1 \leq i \leq k$ and $\phi$ is formulated. The convergence of this algorithm follows immediately from the finite dimension of the state space.

\section{Algorithm 1.}

1. Take $i=1, \Lambda_{\alpha^{(0)}}=\operatorname{ker} \partial h / \partial x$.

2. Find the involutive distribution $\Lambda_{\alpha^{(i)}}=\Lambda_{\beta}$ from (23) and (24) with

$$
\Lambda_{\alpha}=\bigcap_{j=0}^{i-1} \Lambda_{\alpha^{(j)}} .
$$

3. Find the function $\alpha^{(i)}$ by integrating the distribution $\Lambda_{\alpha}^{(i)}$.
4. If $i \geq 2$ and

$$
\bigcap_{j=0}^{i-1} \Lambda_{\alpha^{(j)}} \subseteq \Lambda_{\alpha^{(i)}},
$$

then set $k=i-1$ and go to the next step. Otherwise, set $i=i+1$ and return to Step 2 .

5. Find the function $\phi \circ h$ by integrating the minimal involutive distribution containing

$$
\operatorname{ker} \frac{\partial h}{\partial x}+\bigcap_{j=0}^{i-1} \Lambda_{\alpha^{(j)}} .
$$

Remark 2. In order to minimize the dimension of the transformed model, the function $\alpha^{(i)}$ should contain only the components which are functionally independent of those of the function $\alpha^{(1)} \times \ldots \times \alpha^{(i-1)}$ and other components of $\alpha^{(i)}$. It requires deleting the redundant components of the functions $\alpha^{(i)}, 2 \leq i \leq k$, after obtaining them in Step 3 of the algorithm.

Remark 3. To take the condition (17) into account, it is sufficient to use the involutive distribution

$$
\begin{array}{r}
\Lambda_{\beta} \supseteq \operatorname{span}\left\{\left[\varphi, \lambda_{\alpha}\right], \varphi \in\left\{f, g_{1}, \ldots, g_{p}\right\}, \lambda_{\alpha} \in \Lambda_{\alpha}\right\} \\
+\operatorname{span}\left\{d_{j}\right\}
\end{array}
$$

in spite of (23) in Step 2 of the algorithm.

The implementation of Algorithm 1 requires linear algebra operations, function differentiation and distribution integration. All these computations can be supported by computer algebra systems such as Reduce, Maple, etc.

\section{Linear Case}

The purpose of this section is to make a comparison between the model-free method (Pekpe et al., 2004) and the method proposed above. Recall that the reference method assumes that the system under consideration is specified by linear differential equations with unknown matrices.

Consider the system

$$
\begin{aligned}
& x(t)=F x(t)+G u(t)+D w(t), \\
& y(t)=H x(t)
\end{aligned}
$$

with time invariant matrices $F, E, D, H$ of appropriate dimensions. It is assumed that some or all elements of these matrices are unknown. For the system (25), (26) and $w(t)=0$, the strict feedback form is given by

$$
\begin{aligned}
x_{*}^{(1)}(t) & =L_{*}^{(1)} y(t)+G_{*}^{(1)} u(t), \\
x_{*}^{(i)}(t) & =F_{*}^{(i)} x_{*}^{(i-1)}(t)+L_{*}^{(i)} y(t)+G_{*}^{(i)} u(t), \\
2 \leq i \leq k, & \\
y_{*}(t) & =H_{*}\left(\begin{array}{c}
x_{*}^{(1)}(t) \\
\vdots \\
x_{*}^{(k)}(t)
\end{array}\right),
\end{aligned}
$$


where $G_{*}^{(i)}, L_{*}^{(i)}, 1 \leq i \leq k$, and $H_{*}$ are the matrices of appropriate dimensions. Similarly to the matrices of the initial system (25), (26), some or all elements of these matrices are assumed to be unknown. From (27) and (28), as well as the iterated integrals (8) and the relation (10), we obtain the representation (11) with

$$
\begin{aligned}
& C(t)=H_{*}\left(Z_{1}(t) \quad Z_{2}(t)\right), \\
& W\left(t, t_{*}\right)=\left(\begin{array}{l}
W_{1}\left(t, t_{*}\right) \\
W_{2}\left(t, t_{*}\right)
\end{array}\right) \text {, }
\end{aligned}
$$

where $Z_{1}(t), Z_{2}(t), W_{1}\left(t, t_{*}\right)$ and $W_{2}\left(t, t_{*}\right)$ are given by (29).

It is known that if a pair $(H, F)$ from (25) and (26) is observable, then the system admits an equivalent (i.e., $\phi(y)=y$ ) transformation into a canonical identifiable form (the Kronecker form). The canonical identifiable form is a special case of the strict feedback form with some diagonal matrices $L_{*}^{(i)}, 1 \leq i \leq k$, diagonal matrices $F_{*}^{(i)}, 2 \leq i \leq k$ containing only one or zero on their diagonals, and the index $k \leq n-l+1$. It follows that one can always take, firstly, $k=n-l+1$ in (29) and, secondly, $\Phi_{s}\left(t_{s}\right)=\left(y(t) y\left(t_{1}\right) \ldots y\left(t_{s}\right)\right)$ in (13). Therefore, in the linear system case the computational form for robust redundancy relations is

$$
r\left(t_{s}\right)=\left(y(t) y\left(t_{1}\right) \ldots y\left(t_{s}\right)\right) v\left(t_{s}\right)
$$

with

$v\left(t_{s}\right) \in \operatorname{ker}\left(\begin{array}{llll}W_{1}(t, t) & W_{1}\left(t, t_{1}\right) & \ldots & W_{1}\left(t, t_{s}\right) \\ W_{2}(t, t) & W_{2}\left(t, t_{1}\right) & \ldots & W_{2}\left(t, t_{s}\right)\end{array}\right)$

and, according to (12), the index $s$ is a minimal integer such that

$$
\begin{aligned}
& \operatorname{rank}\left(\begin{array}{llll}
W_{1}(t, t) & W_{1}\left(t, t_{1}\right) & \ldots & W_{1}\left(t, t_{s}\right) \\
W_{2}(t, t) & W_{2}\left(t, t_{1}\right) & \ldots & W_{2}\left(t, t_{s}\right)
\end{array}\right) \\
= & \operatorname{rank}\left(\begin{array}{llll}
W_{1}(t, t) & W_{1}\left(t, t_{1}\right) & \ldots & W_{1}\left(t, t_{s-1}\right) \\
W_{2}(t, t) & W_{2}\left(t, t_{1}\right) & \ldots & W_{2}\left(t, t_{s-1}\right)
\end{array}\right) .
\end{aligned}
$$

Taking into account the size of the vectors $W_{1}\left(t, t_{*}\right)$ and $W_{2}\left(t, t_{*}\right)$ and the inequality $k \leq n-l+1$, it is easy to see that

$$
s \leq(n-l+1)(p+l+1) .
$$

Both the index $s$ and the vector $v\left(t_{s}\right)$ can be calculated on line by handling system inputs and outputs on the time interval $\left[t, t_{s}\right]$. Thus, in the linear case only information about the system dimension (the indices $n, l, p$ ) is required to construct redundancy relations for solving the fault detection problem. Then, in comparison with the former

\footnotetext{
${ }^{2}$ If necessary, one can find specific expressions for these matrices by applying the transformation procedure described in the previous section.
}

model free method (Pekpe et al., 2004), the proposed result allows us to decrease the size of the moving time window that has positive influence on the minimization of the fault detection delay. Indeed, a large size of the moving time window in the former method is caused by the need to ignore the effect of the free system movement due to an unknown initial state because the techniques of the initial state elimination are not used. In the proposed method, the unknown initial state vectors are included into the matrix $Z_{2}(t)$ and the residual is insensitive to the value of this matrix by construction.

The solution of the fault isolation problem requires more information about the system. Indeed, constructing the redundancy relations for fault isolation needs fulfilling (17) for some faults and its violation for others. As a result, the necessary condition for distinguishing the faults caused by $w_{i}(t) \neq 0$ and $w_{j}(t) \neq 0$ consists in the linear independence of the appropriate columns of the matrix $D$. Accordingly, to isolate all faults, one needs fulfilling the rank condition

$$
\operatorname{rank} D=q
$$

Assume that the available information about the system is sufficient to conclude about the possible isolability of the faults. The solution of the fault isolation problem needs using the general form of redundancy relations (13). Constructing the redundancy relations amounts to finding the function $\phi(y)$ and index $k$. As in the general case, the last problem is solved by using Algorithm 1. Then, as soon as in the linear case all distributions contain only constant vectors, the equality (24) holds automatically and $\left[\varphi, \lambda_{\alpha}\right]=F \lambda_{\alpha}$ for $\varphi=F x$.

\section{Nonlinear Example}

Consider the model described by (1) and (2) with the functions

$$
f(x)=\left(\begin{array}{c}
\vartheta_{1} x_{1}^{2} \\
\vartheta_{2} x_{1} x_{2} \\
\vartheta_{3} x_{4}+\vartheta_{4} x_{1} x_{2} \\
x_{3} \\
x_{1} x_{2}
\end{array}\right)
$$

$$
\begin{gathered}
g(x)=d(x)=\left(\begin{array}{ccc}
\vartheta_{5} & \vartheta_{5} & 0 \\
\vartheta_{6} / x_{1} & -\vartheta_{6} / x_{1} & \vartheta_{7} / x_{1} \\
0 & 0 & \vartheta_{8} \\
0 & 0 & 0 \\
0 & 0 & 0
\end{array}\right), \\
h(x)=\left(\begin{array}{c}
x_{1} \\
x_{4} \\
x_{5}
\end{array}\right) .
\end{gathered}
$$




$$
\begin{aligned}
& Z_{1}(t)=\left(\begin{array}{cccccccc}
L^{(1)} & G^{(1)} & 0 & \cdot & . & \cdot & . & \ldots \\
L^{(2)} & G^{(2)} & F^{(2)} L^{(1)} & F^{(2)} G^{(1)} & 0 & . & . & \ldots \\
\vdots & \vdots & \vdots & \vdots & \vdots & \vdots & \ddots & \vdots \\
L^{(k)} & G^{(k)} & F^{(k)} L^{(k-1)} & F^{(k)} G^{(k-1)} & . & . & . & F^{(k)} L^{(k-1)} \ldots F^{(2)} L^{(1)}
\end{array}\right. \\
& \left.\begin{array}{c}
0 \\
0 \\
F^{(k)} L^{(k-1)} \ldots F^{(2)} G^{(1)}
\end{array}\right) \\
& Z_{2}(t)=\left(\begin{array}{ccccc}
x_{*}^{(1)}(t) & 0 & \ldots & . & 0 \\
x_{*}^{(2)}(t) & F^{(2)} x_{*}^{(1)}(t) & 0 & \ldots & 0 \\
\vdots & \vdots & \vdots & \ddots & \vdots \\
x_{*}^{(k)}(t) & F^{(k)} x_{*}^{(k-1)}(t) & F^{(k)} F^{(k-1)} x_{*}^{(k-2)}(t) & \ldots & F^{(k)} F^{(k-1)} \ldots F^{(2)} x_{*}^{(1)}(t)
\end{array}\right)
\end{aligned}
$$

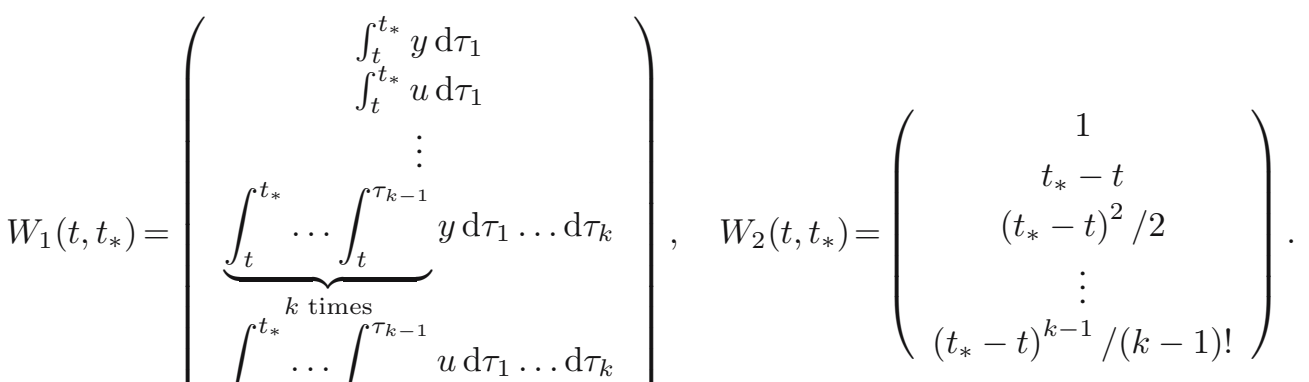

The above equations constitute a simplified model of the underwater vehicle CR-01 moving in a vertical plane obtained under the assumption about a small velocity and a small angle of the trajectory from the available vehicle model (Shumsky, 2006) by the Tailor series expansion of trigonometric functions while neglecting higher order terms. Model variables have the following sense: $x_{1}$ is the velocity, $x_{2}$ is the angle of the trajectory, $x_{4}$ and $x_{3}$ are the trim and its time derivative, respectively, $x_{5}$ is the depth. Model coefficients $\vartheta_{1}, \ldots, \vartheta_{8}$ characterize the masses and inertia denoted by $m_{x}, m_{y}$ and $J_{z}$ with additions taken with respect to the appropriate coordinates as well as the structural features of the vehicle denoted by $r_{0 x}, r_{1 x}, r_{0 x}, r_{2 y}, r_{0 x}, M_{0}, \hat{m}_{z}, \delta$ (for details, see Shumsky, 2006). The links between the model coefficients and

\footnotetext{
${ }^{3}$ This simplification is caused only by the necessity to make the model compact and usable in the framework of a journal paper to illustrate the details of designing redundancy relations. The details of the simplification are omitted because they do not relate to the method under consideration.
}

vehicle parameters are given as follows:

$$
\begin{aligned}
\vartheta_{1} & =\frac{\rho}{2 m_{x}}\left(r_{0 x}+r_{1 x}+r_{2 x}\right), & \vartheta_{2} & =\frac{\rho}{2 m_{y}} r_{2 y}, \\
\vartheta_{3} & =\frac{M_{0}}{J_{z}}, & \vartheta_{4} & =-\frac{\rho \hat{m}_{z}}{J_{z}}, \\
\vartheta_{5} & =\frac{\cos \delta}{m_{x}}, & \vartheta_{6} & =\frac{\sin \delta}{m_{y}}, \\
\vartheta_{7} & =\frac{1}{m_{y}}, & \vartheta_{8} & =\frac{1}{J_{z}},
\end{aligned}
$$

where $\rho$ is the water density. All the model coefficients are assumed below to be constant but unknown. The inputs $u_{1}, u_{2}$, and $u_{3}$ are the forces of the upper and bottom stern thrusters, and the vertical bow thruster, respectively.

Based on this model, the solution to two problems is demonstrated. The first problem is to construct a redundancy relation for fault detection, while the second deals with fault isolation.

Consider the solution to the first problem. Let us begin by determining the functions $\alpha^{(i)}, 1 \leq i \leq k$ and $\phi$. Calculating the functions $\alpha^{(i)}, 1 \leq i \leq k$ according to 
Steps 1-4 of Algorithm 1 implies the following results:

$$
\Lambda_{\alpha(0)}=\operatorname{span}\left\{\left(\begin{array}{ll}
0 & 0 \\
1 & 0 \\
0 & 1 \\
0 & 0 \\
0 & 0
\end{array}\right)\right\}
$$

$\operatorname{span}\left\{\left[\varphi, \lambda_{\alpha}\right], \varphi \in\left\{f, g_{1}, g_{2}, g_{3}\right\}, \Lambda_{\alpha^{(0)}}\right\}$

$$
=\operatorname{span}\left\{\left(\begin{array}{cc}
0 & 0 \\
\vartheta_{2} x_{1} & 0 \\
\vartheta_{4} x_{1} & 0 \\
0 & 1 \\
x_{1} & 0
\end{array}\right)\right\}
$$

It is easy to check that the last distribution is involutive and satisfies (24). It allows us to take this distribution as $\Lambda_{\alpha^{(1)}}$. Its integration gives

$$
\alpha^{(1)}(x)=\left(\begin{array}{c}
x_{1} \\
x_{2}-\vartheta_{2} x_{5} \\
x_{3}-\vartheta_{4} x_{5}
\end{array}\right) \text {. }
$$

Then $\Lambda_{\alpha^{(0)}} \cap \Lambda_{\alpha^{(1)}}=\operatorname{span}\{(0)\}$ and

$$
\begin{array}{r}
\operatorname{span}\left\{\left[\varphi, \lambda_{\alpha}\right], \varphi \in\left\{f, g_{1}, g_{2}, g_{3}\right\}, \lambda_{\alpha} \in \Lambda_{\alpha^{(0)}} \cap \Lambda_{\alpha^{(1)}}\right\} \\
=\operatorname{span}\{(0)\}=\Lambda_{\alpha^{(2)}} .
\end{array}
$$

Integrating this distribution gives $\operatorname{col}\left(x_{1}, x_{2}, x_{3}, x_{4}, x_{5}\right)$. Excluding the redundant components $x_{1}, x_{2}, x_{3}$ dependent on the components of $\alpha^{(1)}$ and other components of the sought function, we obtain

$$
\alpha^{(2)}(x)=\left(\begin{array}{l}
x_{4} \\
x_{5}
\end{array}\right) .
$$

Since $\Lambda_{\alpha^{(1)}} \cap \Lambda_{\alpha^{(2)}}=\operatorname{span}\{(0)\}$, we have $\Lambda_{\alpha^{(1)}} \cap$ $\Lambda_{\alpha^{(2)}} \subseteq \Lambda_{\alpha^{(3)}}$ independently of $\Lambda_{\alpha^{(3)}}$ and hence $k=2$.

According to Step 5 of Algorithm 1, the function $\phi \circ h$ is obtained from the distribution

$$
\operatorname{ker} \frac{\partial h}{\partial x}+\left(\Lambda_{\alpha^{(1)}} \cap \Lambda_{\alpha^{(2)}}\right)=\operatorname{span}\left\{\left(\begin{array}{ll}
0 & 0 \\
1 & 0 \\
0 & 1 \\
0 & 0 \\
0 & 0
\end{array}\right)\right\}
$$

in the form

$$
\phi \circ h=\left(\begin{array}{l}
x_{1} \\
x_{4} \\
x_{5}
\end{array}\right)
$$

that gives

$$
\phi=\left(\begin{array}{l}
y_{1} \\
y_{2} \\
y_{3}
\end{array}\right)
$$

Using the above functions $\alpha^{(1)}, \alpha^{(2)}$ and $\phi$, the description of the transformed model is obtained from (18) and (19) in the form

$$
\begin{aligned}
f_{*}^{(1)}(y) & =\left(\begin{array}{c}
\vartheta_{1} y_{1}^{2} \\
0 \\
\vartheta_{3} y_{2}
\end{array}\right), \\
g_{*}^{(1)}(y) & =\left(\begin{array}{ccc}
\vartheta_{5} & \vartheta_{5} & 0 \\
\vartheta_{6} / y_{1} & -\vartheta_{6} / y_{1} & \vartheta_{7} / y_{1} \\
0 & 0 & \vartheta_{8}
\end{array}\right), \\
f_{*}^{(2)}\left(y, x_{*}^{(1)}\right) & =\left(\begin{array}{c}
x_{* 3}^{(1)}+\vartheta_{4} y_{3} \\
y 1\left(x_{* 2}^{(1)}+\vartheta_{2} y_{3}\right)
\end{array}\right), \\
g_{*}^{(2)}\left(y, x_{*}^{(1)}\right) & \left.=0, \begin{array}{c}
x_{* 1}^{(1)} \\
x_{* 1}^{(2)} \\
x_{* 2}^{(2)}
\end{array}\right) . \\
h_{*}\left(x_{*}^{(1)}, x_{*}^{(2)}\right) & =
\end{aligned}
$$

Then, using (8)-(10), it is easy to convert the transformed model to the input-output description

$$
\begin{aligned}
y_{1}\left(t+t_{*}\right)= & \int_{t}^{t_{*}}\left(\vartheta_{1} y_{1}^{2}+\vartheta_{5}\left(u_{1}+u_{2}\right)\right) \mathrm{d} \tau_{1}+x_{* 1}^{(1)}(t), \\
y_{2}\left(t+t_{*}\right)= & \int_{t}^{t_{*}}\left(\vartheta_{4} y_{3}+\int_{t}^{\tau_{1}}\left(\vartheta_{3} y_{2}+\vartheta_{8} u_{3}\right) \mathrm{d} \tau_{2}\right. \\
& \left.+x_{* 3}^{(1)}\right) \mathrm{d} \tau_{1}+x_{* 1}^{(2)}(t), \\
y_{3}\left(t+t_{*}\right)= & \int_{t}^{t_{*}} y_{1}\left(\int_{t}^{\tau_{1}}\left(\vartheta_{6}\left(u_{1}-u_{2}\right) / y_{1}+\vartheta_{7} u_{3} / y_{1}\right)\right. \\
& \left.\times \mathrm{d} \tau_{2}+x_{* 2}^{(1)}(t)+\vartheta_{2} y_{3}\right) \mathrm{d} \tau_{1}+x_{* 2}^{(2)}(t)
\end{aligned}
$$

and, after this, to the matrix description with the matrix

$C(t)$

$$
=\left(\begin{array}{ccccccccc}
\vartheta_{1} & \vartheta_{5} & 0 & 0 & 0 & 0 & 0 & x_{* 1}^{(1)}(t) & 0 \\
0 & 0 \vartheta_{4} & \vartheta_{3} & \vartheta_{8} & 0 & 0 & x_{* 1}^{(2)}(t) & x_{* 3}^{(1)}(t) \\
0 & 0 \vartheta_{2} & 0 & 0 & \vartheta_{6} & \vartheta_{7} & x_{* 2}^{(2)}(t) & x_{* 2}^{(1)}(t)
\end{array}\right),
$$


and the vector

$$
\begin{gathered}
W\left(t, t_{*}\right) \\
=\operatorname{col}\left(\int_{t}^{t_{*}} y_{1}^{2} \mathrm{~d} \tau_{1}, \int_{t}^{t_{*}}\left(u_{1}+u_{2}\right) \mathrm{d} \tau_{1}, \int_{t}^{t_{*}} y_{3} \mathrm{~d} \tau_{1},\right. \\
\quad \int_{t}^{t_{*}} \int_{t}^{\tau_{1}} y_{2} \mathrm{~d} \tau_{2} \mathrm{~d} \tau_{1}, \int_{t}^{t_{*}} \int_{t}^{\tau_{1}} u_{3} \mathrm{~d} \tau_{2} \mathrm{~d} \tau_{1}, \\
\quad \int_{t}^{t_{*}} y_{1} \int_{t}^{\tau_{1}}\left(\left(u_{1}-u_{2}\right) / y_{1}\right) \mathrm{d} \tau_{2} \mathrm{~d} \tau_{1}, \\
\left.\int_{t}^{t_{*}} y_{1} \int_{t}^{t_{*}}\left(u_{3} / y_{1}\right) \mathrm{d} \tau_{2} \mathrm{~d} \tau_{1}, 1, t_{*}-t\right) .
\end{gathered}
$$

The vector $W\left(t, t_{*}\right)$ and the function $\phi$ are used for residual generation according to (13). Then fulfilling $\left(\partial \alpha^{(1)} / \partial x\right) d_{j} \neq 0,1 \leq j \leq 3$ indicates the possibility to detect all faults.

To confirm the design, simulation results are given in Figs. 2-4. In simulations every fault was specified as a $30 \%$ reduction in the appropriate thruster force appearing at $t=80 \mathrm{~s}, t=110 \mathrm{~s}$ and $t=130 \mathrm{~s}$ for the upper stern, bottom stern and vertical bow thrusters, respectively. Faults were retained to the end of simulations. Moveover, $u_{1}(t)=u_{2}(t)=100[\mathrm{n}]$ and $u_{3}(t)=100[\mathrm{n}]$ if $t \leq 30[\mathrm{~s}] ; u_{3}(t)=0[\mathrm{n}]$ if $30 \leq t \leq 120[\mathrm{~s}], t>170[\mathrm{~s}]$, and $u_{3}(t)=-100[\mathrm{n}]$ if, $120[\mathrm{~s}]<t \leq 170[\mathrm{~s}]$. The sampling period was taken as ${ }_{\Delta} t=2[\mathrm{~s}]$. From Figs. 2-4 it follows that all faults are successfully detected.

As for the fault isolation problem, let us touch only upon some features of finding the functions $\alpha^{(i)}$ and $\phi$. Concentrate on constructing the redundancy relation which guarantees the insensitivity of the residual to the fault in the vertical bow thruster. In this case, according to Remark 3, the distribution $\operatorname{span}\left\{d_{3}\right\}$ is involved in determining the functions $\alpha^{(i)}$. Steps $1-4$ of Algorithm 1 result in

$$
\begin{aligned}
& \Lambda_{\alpha(1)}=\operatorname{span}\left\{\left(\begin{array}{ccc}
0 & 0 & 0 \\
\vartheta_{2} x_{1} & 0 & \vartheta_{7} / x_{1} \\
\vartheta_{4} x_{1} & 0 & \vartheta_{8} \\
0 & 1 & 0 \\
x_{1} & 0 & 0
\end{array}\right)\right\}, \\
& =\left(\begin{array}{c}
x_{1} x_{1} x_{2}-\vartheta_{7} x_{3}+x_{5}\left(\vartheta_{4} \vartheta_{7}-\vartheta_{2} \vartheta_{8} x_{1}\right)
\end{array}\right),
\end{aligned}
$$

$\Lambda_{\alpha^{(2)}}$

$$
=\operatorname{span}\left\{\left(\begin{array}{ccc}
0 & 0 & 0 \\
\left(\vartheta_{1}-\vartheta_{2}\right) \vartheta_{7} & \vartheta_{5} \vartheta_{7} / x_{1}^{2} & \vartheta_{7} / x_{1} \\
-\vartheta_{4} \vartheta_{7} x_{4} / x_{1} & 0 & \vartheta_{8} \\
\vartheta_{8} & 0 & 0 \\
\vartheta_{7} & 0 & 0
\end{array}\right)\right\} \text {, }
$$

$$
\alpha^{(2)}(x)=\vartheta_{7} x_{4}-\vartheta_{8} x_{5}, \quad k=2 .
$$

In Step 5 we obtain

$$
\operatorname{ker} \frac{\partial h}{\partial x}+\Lambda_{\alpha^{(1)}} \cap \Lambda_{\alpha^{(2)}}=\operatorname{span}\left\{\left(\begin{array}{ccc}
0 & 0 & 0 \\
1 & 0 & 0 \\
0 & 1 & 0 \\
0 & 0 & \vartheta_{8} \\
0 & 0 & \vartheta_{7}
\end{array}\right)\right\} \text {. }
$$

It results in

$$
\phi \circ h=\left(\begin{array}{c}
x_{1} \\
\vartheta_{7} x_{4}-\vartheta_{8} x_{5}
\end{array}\right),
$$

which gives

$$
\phi=\left(\begin{array}{c}
y_{1} \\
\vartheta_{7} y_{2}-\vartheta_{8} y_{3}
\end{array}\right) .
$$

But as soon as the function $\phi$ should be independent of unknown coefficients, one finally has $\phi=y_{1}$.
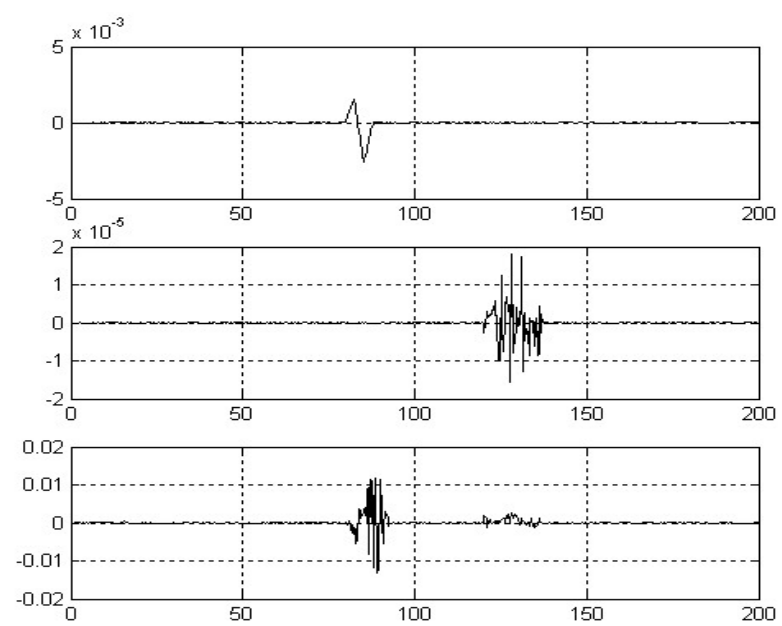

Fig. 2. Three-component residual vector behavior under a fault in the upper stern thruster occurring at $t=80[\mathrm{~s}]$.

\section{Conclusion}

In this paper, a novel method has been proposed for constructing the redundancy relations for robust FDI in nonlinear uncertain systems. The method involves a nonlinear transformation of the initial system model into a strict feedback form. Existence conditions for the transformation have been formulated and an algorithm has been developed. The implementation of this algorithm may be supported by existing computer algebra systems.

For linear systems, the proposed method does not require an explicit model. Moreover, in comparison with the former model-free method (Pekpe et al., 2004), it allows 

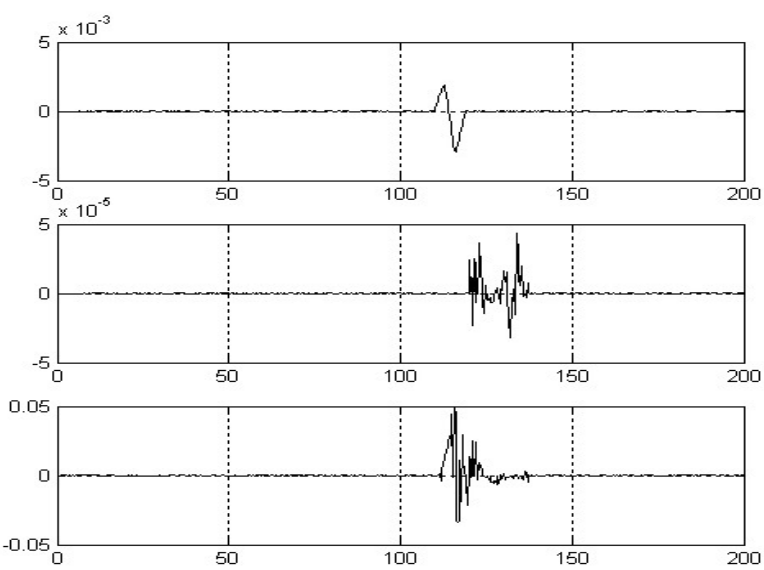

Fig. 3. Three-component residual vector behavior under a fault in the bottom stern thruster occurring at $t=110[\mathrm{~s}]$.
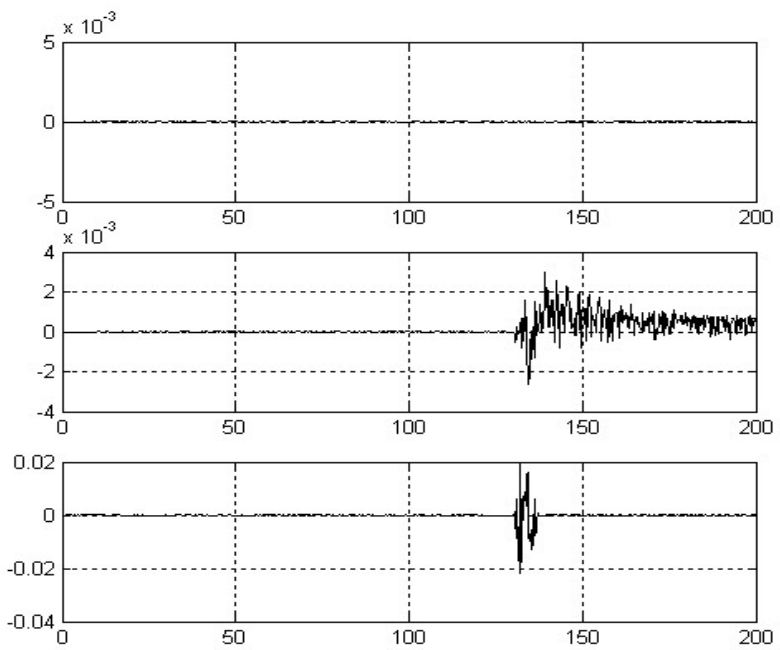

Fig. 4. Three-component residual vector behavior under a fault in the vertical bow thruster occurring at $t=130[\mathrm{~s}]$.

us to decrease the size of the moving time window. As a result, the fault detection delay is minimized. Additionally, FDI becomes possible not only in sensors, but in a plant and actuators, too.

The existence condition for the transformation into the strict feedback form imposes the restriction on the class of nonlinear systems under diagnosis. But it is even worse to note that redundancy relations are equivalent to some nonlinear observer-based residual generator with dead-beat characteristics. Known design methods which guarantee the stability of the nonlinear observer involve a perfect or partial transformation of the initial system model into the so-called nonlinear observer canonical form. It can be shown that the last form is only a special case of the strict feedback form. Therefore, the restriction of the proposed method is less strict than the conventional one.

\section{Acknowledgment}

This work was supported by the Russian Found of Basic Research, Grant No. 05-08-18240.

\section{References}

Chow E. Y. and A.S. Willsky. (1984): Analytical redundancy and the design of robust failure detection systems. IEEE Transactions on Automatic Control, Vol. AC-29, No. 7, pp. 603-614.

Comtet-Varga G., C. Christophe, V. Cocquempot and M. Staroswiecki. (1999): F.D.I. For the induction motor using elimination theory. Proceedings of the European Control Conference, Karlsruhe, Germany, (on CD-ROM)

Cox D., Little J. and D. O'Shea. (1992): Ideals, Varieties and Algorithms. New York: Springer.

De Persis C. and A. Isidori. (2001): A geometric approach to nonlinear fault detection and isolation. IEEE Transactions on Automatic Control, Vol. AC-46, No. 6, pp. 853-865.

De Persis C. and A. Isidori. (2002): Fault detection filters. International Journal of Robust and Nonlinear Control, Vol. 12, No. 6, pp. 729-747.

Ding X. and P.M. Frank. (1993): An adaptive observer-based fault detection scheme for nonlinear dynamic systems. Proceedings of the 12-th IFAC World Congress, Sydney, Australia, Vol. 8, pp. 307-310

Diop S. (1991): Elimination in control theory. - Mathematics of Control, Signals, and Systems, Vol. 14, No. 1, pp. 459-474

Frank P.M. (1990): Fault diagnosis in dynamic systems using analytical and knowledge-based redundancy - A survey and some new results. Automatica, Vol. 26, No 3, pp. 459474.

Gertler J. and M.M. Kunwer. (1993): Optimal residual decoupling for robust fault diagnosis. Proceedings of the International Conference Tooldiag'93, Toulouse, France, pp. 436-452.

Isermann R. (1984): Process fault detection based on modeling and estimation methods: A survey. Automatica, Vol. 20, No. 4, pp. 387-404.

Isidori A. (1989): Nonlinear Control Systems. Berlin: Springer.

Lou X.C., Willsky A.S. and G.L. Verghese. (1986): Optimally robust redundancy relations for failure detection in uncertain systems. Automatica, Vol. 22, No. 3, pp. 333-344.

Massoumnia M. A. (1986): A geometric approach to the synthesis of failure detection filters. IEEE Transactions on Automatic Control, Vol. AC-31, No. 9, pp. 839-846.

Massoumnia M. A., G. C. Verghese and A. S. Willsky. (1989): Failure detection and identification. IEEE Transactions on Automatic Control, Vol. AC-34, No. 3, pp. 316-323.

Medvedev A. (1994): Parity space method: A continuous time approach. Proceedings of the American Control Conference, Baltimore, Vol. 1, pp. 662-665.

Mironovskii L.A. (1980): Functional diagnosis of dynamic systems. Automation and Remote Control, Vol. 41, No. 8, pp. 1122-1143. 
Park J., G. Rizzoni and W.B. Ribbens. (1994): On the representation of sensor faults in fault detection filters. Automatica, Vol. 30, No. 11, pp. 1793-1795.

Patton R. and S. Kangethe. (1989): Robust fault diagnosis using eigenstructure assignment of observers. In: Fault Diagnosis in Dynamic Systems. Theory and Application (Patton R.J., Frank P.M., Clark R.N. N.Y., Eds.), Englewood Cliffs: Prentice Hall, pp. 99-154.

Pekpe, K. M., G. Mourot and J. Ragot. (2004): Subspace method for sensors fault detection and isolation - application to grinding circuit monitoring. Proceedings of the 11th IFAC Symposium on Automation in Mining, Mineral and Metal Processing, Nancy, France.

Ritt J.F. (1950): Differential Algebra. New York, American Math. Society.

Seliger R. and P.M. Frank. (1991): Robust component fault detection and isolation in nonlinear dynamic systems using nonlinear unknown input observers. Proceedings of the IFAC Symposium SAFEPROCESS'91, Baden-Baden, Germany, pp. 313-318.

Shumsky, A.Ye. (1991): Fault isolation in nonlinear systems via functional diagnosis. Automation and Remote Control, Vol. 52, No. 12, pp. 1759-1765.

Shumsky, A.Ye. (1992): Diagnosis of parametric errors in dynamic objects by the hypothesizes testing method. Avtomatika i telemehanika, Vol. 53, No. 10, pp. 171-177 (in Russian).

Shumsky, A.Ye. (2002): Robust analytical redundancy relations for fault diagnosis in nonlinear systems. Asian Journal of Control, Vol. 4, No 2, pp. 159-170.

Shumsky, A. Ye. and A. N. Zhirabok. (2006): Nonlinear diagnostic filter design: Algebraic and geometric points of view. International Journal of Applied Mathematics and Computer Science, Vol. 16, No. 1, pp. 115-127.

Shumsky, A. Ye. (2006): Fault diagnosis of sensors in autonomous underwater vehicle: Adaptive quasi-linear parity relations method. Proceedings of the International Symposium SAFEPROCESS, Beijing, PR China, pp. 415-520.

\section{Appendix}

Proof of Lemma 1. (Sufficiency) Assume that there exist functions $\alpha^{(i)}, 1 \leq i \leq k$, and $\phi$ satisfying (4) and (5), respectively. Differentiating both the sides of (4) with respect to time gives

$$
x_{*}^{(i)}=\frac{\partial \alpha^{(i)}}{\partial x}(f(x)+g(x) u), 1 \leq i \leq k .
$$

Also, substituting (4) and (5) into (6) gives

$$
\begin{aligned}
x_{*}^{(1)}= & f_{*}^{(1)}(h(x))+g_{*}^{(1)}(h(x)) u, \\
x_{*}^{(i)}= & f_{*}^{(i)}\left(\alpha^{(1)}(x), \ldots, \alpha^{(i-1)}(x), h(x)\right) \\
& +g_{*}^{(i)}\left(\alpha^{(1)}(x), \ldots, \alpha^{(i-1)}(x), h(x)\right) u, \\
& \quad 2 \leq i \leq k .
\end{aligned}
$$

Taking into account that for every $i, 1 \leq i \leq k$, the left-hand sides of the above equalities coincide, for $i=1$ one has

$$
f_{*}^{(1)}(h(x))+g_{*}^{(1)}(h(x)) u=\frac{\partial \alpha^{(1)}}{\partial x}(f(x)+g(x) u)
$$

and

$$
\begin{aligned}
f_{*}^{(i)} & \left(\alpha^{(1)}(x), \ldots, \alpha^{(i-1)}(x), h(x)\right) \\
& +g_{*}^{(i)}\left(\alpha^{(1)}(x), \ldots, \alpha^{(i-1)}(x), h(x)\right) u \\
& =\frac{\partial \alpha^{(i)}}{\partial x}(f(x)+g(x) u) \quad \text { for } \quad 2 \leq i \leq k .
\end{aligned}
$$

Assuming that the above equalities are true for every $u \in U$, we obtain (18). Then (19) follows immediately from (2), (4), (5) and (7).

(Necessity) Let us show that the description (6) and (7) of the strict feedback form follows immediately from (18) and (19). To this end, assume that (18) holds. Let $x_{*}^{(i)}=$ $\alpha^{(i)}(x)$ and, therefore, $x_{*}^{(i)}=\mathrm{d} \alpha^{(i)}(x) / \mathrm{d} t, 1 \leq i \leq k$. Taking into account (2), we obtain

$$
\begin{gathered}
f_{*}^{(1)}(y)+g_{*}^{(1)}(y) u \\
=f_{*}^{(1)}(h(x))+g_{*}^{(1)}(h(x)) u \\
=\frac{\partial \alpha^{(1)}}{\partial x}(f(x)+g(x) u) \\
=\frac{\mathrm{d} \alpha^{(1)}(x)}{\mathrm{d} t}=x_{*}^{(1)}, \\
f_{*}^{(i)}\left(x_{*}^{(1)}, \ldots, x_{*}^{(i-1)}, y\right)+g_{*}^{(i)}\left(x_{*}^{(1)}, \ldots, x_{*}^{(i-1)}, y\right) u \\
=f_{*}^{(i)}\left(\alpha^{(1)}(x), \ldots, \alpha^{(i-1)}(x), h(x)\right) \\
\quad+g_{*}^{(i)}\left(\alpha^{(1)}(x), \ldots, \alpha^{(i-1)}(x), h(x)\right) u \\
=\frac{\partial \alpha^{(1)}}{\partial x}(f(x)+g(x) u)=x_{*}^{(i)} .
\end{gathered}
$$

Equations (6) follow then immediately. Assume that (19) holds. Taking $y_{*}=\phi(y)$, we obtain

$$
\begin{aligned}
y_{*} & =\phi(y)=\phi(h(x))=h_{*}\left(\alpha^{(1)}(x), \ldots, \alpha^{(k)}(x)\right) \\
& =h_{*}\left(x^{(1)}, \ldots, x^{(k)}\right)
\end{aligned}
$$

which results in (7).

Proof of Theorem 1. (Sufficiency) Let us show that (20) and (21) follow from (18) and (19), respectively. According to the definitions of the partial preordering relation and the binary operation, from (18) we can write

$$
\begin{aligned}
& h \leq \frac{\partial \alpha^{(1)}}{\partial x} f, \quad h \leq \frac{\partial \alpha^{(1)}}{\partial x} g_{j}, \\
& 1 \leq j \leq p, \quad \text { for } \quad i=1
\end{aligned}
$$


and

$$
\begin{aligned}
& h \times \alpha^{(1)} \times \ldots \times \alpha^{(i-1)} \leq \frac{\partial \alpha^{(i)}}{\partial x} f, \\
& h \times \alpha^{(1)} \times \ldots \times \alpha^{(i-1)} \leq \frac{\partial \alpha^{(i)}}{\partial x} g_{j}, \quad 1 \leq j \leq p,
\end{aligned}
$$

for $2 \leq i \leq k$.

Then, from these functional inequalities, we obtain (20) according to the definition of the binary relation $\Delta$. The functional inequality (21) follows from (19) according to the definitions of the partial preordering relation and the binary operation.

(Necessity) According to the definition of the binary relation $\Delta$, from (20) we can write

$$
h \leq \frac{\partial \alpha^{(1)}}{\partial x} f, \quad h \leq \frac{\partial \alpha^{(1)}}{\partial x} g_{j}, \quad 1 \leq j \leq p,
$$

for $i=1$ and

$$
\begin{aligned}
& h \times \alpha^{(1)} \times \ldots \times \alpha^{(i-1)} \leq \frac{\partial \alpha^{(i)}}{\partial x} f, \\
& h \times \alpha^{(1)} \times \ldots \times \alpha^{(i-1)} \leq \frac{\partial \alpha^{(i)}}{\partial x} g_{j}, \quad 1 \leq j \leq p
\end{aligned}
$$

for $2 \leq i \leq k$.

Then, according to the definitions of the partial preordering relation, there exist vector functions, denoted by $f_{*}^{(i)}$ and $g_{*}(i), 1 \leq i \leq k$, such that (18) holds. Similarly, the existence of the vector function, denoted by $h_{*}$ and satisfying (19), follows from (21).

Received: 5 March 2007

Revised: 7 July 2007 
